\title{
Identifying Novel Polar Distortion Modes in Engineered Magnetic Oxide Superlattices
}

Saurabh Ghosh, ${ }_{1}^{1}$ Amber Choquette, ${ }^{2}$ Steve May, ${ }^{2}$ Mark P. Oxley, ${ }^{3}$ Andrew R. Lupini, ${ }^{3}$ Sokrates T. Pantelides, ${ }^{1}$ Albina Y. Borisevich ${ }^{3}$

1. Department of Physics and Astronomy, Vanderbilt University, Nashville, TN, USA

2. Department of Materials Science and Engineering, Drexel University, Philadelphia, PA, USA

3. Materials Science and Technology Division and Institute for Functional Imaging of Materials, Oak Ridge National Laboratory, Oak Ridge, TN, USA

One of the fundamental topics in electron microscopy research is to understand the properties that arise at heterointerfaces. Scanning transmission electron microscopes, with sub $\AA$ spatial resolution and pm level precision, are indispensable in these studies [1]. For the case of short period artificial superlattices, it is especially important since the structure and chemistry of every layer can be distinct. With quantitative STEM, we can determine atomic positions, local distortions and octahedral tilts in for every unit cell, providing ideal comparison to first principles studies. Electron energy loss spectroscopy provides additional chemical and electronic structure information.

Oxide superlattices of the type $\left(\mathrm{RFeO}_{3}\right)_{\mathrm{n}} /\left(\mathrm{R}^{\prime} \mathrm{FeO}_{3}\right)_{\mathrm{n}}$, where $\mathrm{R}, \mathrm{R}^{\prime}$ are different rare earth cations, have been theoretically predicted to have high linear magnetoelectric coefficients, making them attractive for applications [2]. However, growing these superlattices experimentally poses challenges due to close chemical nature of the two cations. Additionally, magnetoelectric behavior is predicted to have a complicated coupling mechanism to the local structural distortions, in particular being connected to "improper" polar distortion modes. Analyzing this behavior experimentally will require complicated symmetry analysis of the microscopy data.

Superlattices $\left(\mathrm{LaFeO}_{3}\right)_{\mathrm{n}} /\left(\mathrm{EuFeO}_{3}\right)_{\mathrm{n}}$ were grown by Molecular Beam Epitaxy for $\mathrm{n}=2,3,4,5$ on $\mathrm{DyScO}_{3}$ substrate. A representative STEM image for $n=3$ is shown in Fig.1(a). We observe the formation of a regular superlattice with well separated La and Eu regions (see Fig 1(c)) and periodic changes in the structure (Fig. 1(b)). Chemical separation has also been confirmed by EELS studies (not shown).

In order to enable symmetry mode analysis of the microscopy data for the purposes of comparison with theoretical studies, we are using CBED pattern arrays (4D STEM) coupled with extensive image simulations. Prospects and potential of symmetry mode analysis for studies of oxides will be discussed [3].

References:

[1] SJ Pennycook et al., MRS Bull. (2015).

[2] S. Ghosh et al., Phys. Rev. B (2015).

[3] Research is supported in part by Laboratory Director Research and Development Fund (MPO, AYB, ARL) and DOE Grant No. DE-FG02-09ER46554 (SG, STP). 


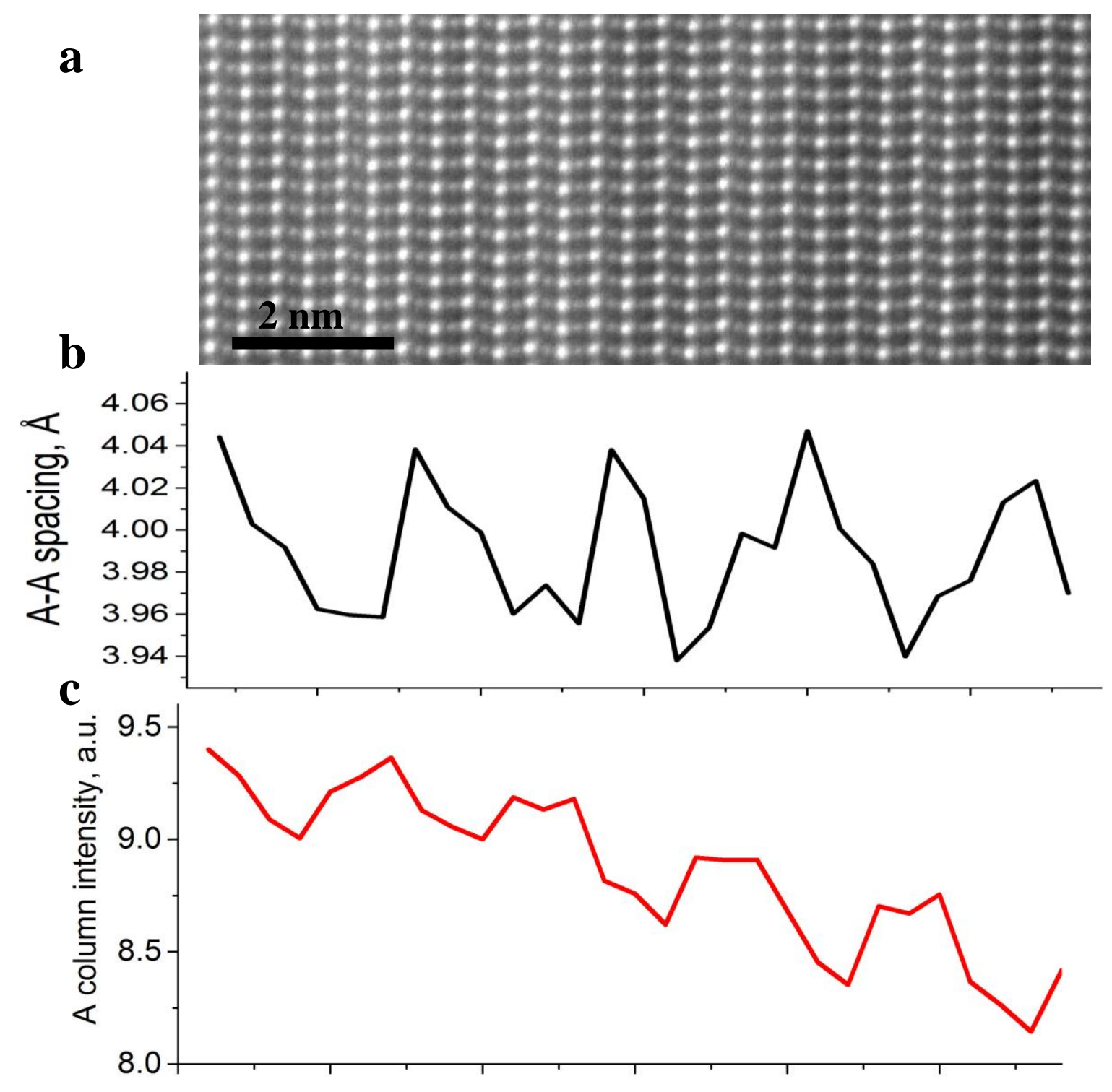

Figure 1. (a) HAADF STEM image of $\left(\mathrm{LaFeO}_{3}\right)_{3} /\left(\mathrm{EuFeO}_{3}\right)_{3}$ superlattice (b) profile of A-A cation spacings computed from the image in (a); (c) profile of A column intensity from the image in (a). Preliminary data suggests that superlattice components are well separated chemically, resulting in periodic changes in structure. 